\title{
Miniature Interferometer Terminals for Earth Surveying
}

Charles C. Counselman III and Irwin I. Shapiro

Department of Earth and Planetary Sciences

Massachusetts Institute of Technology, Cambridge, Massachusetts 02139

Abstract. A system of miniature radio interferometer terminals is proposed for the measurement of vector baselines with uncertainties ranging from the millimeter to the centimeter level for baseline lengths ranging, respectively, from a few to a few hundred kilometers. Each terminal would have no moving parts, could be packaged in a volume of less than $0.1 \mathrm{~m}^{3}$, and would operate unattended. These units would receive radio signals from low-power $(<10 \mathrm{w})$ transmitters on Earthorbiting satellites. The baselines between units could be determined virtually instantaneously and monitored continuously as long as at least four satellites were visible simultaneously. Acquisition of the satellite signals by each terminal would require about one minute, but about one second of signal integration, and the collection of only a few kilobits of data from two receiving units would suffice to determine a baseline. Different baseline lengths, weather conditions, and desired accuracies would, in general, dictate different integration times.

The system proposed here could be used to monitor the regional accumulation and release of strain preceding, following, and even during earthquakes. The terminals could. be deployed in arrays of various dimensions and densities. Their use could also include monitoring variations in transcontinental and intercontinental baselines, but with reduced accuracy. Comparisons with other systems proposed for extensive measurements of regional baseline vectors appear to favor this interferometric approach.

\section{Introduction}

The technique of very-long-baseline interferometry (VLBI) is only a decade old and barely approaching adolescence. Nonetheless this radio interferometric method has seen broad application, especially in astronomy. In geodetic applications, the demonstrated level of repeatability of baseline-length determinations ranges from $\sim 3 \mathrm{~mm}$ for $\sim 1 \mathrm{~km}$ distances (Rogers et al. , 1978) to $\sim 3 \mathrm{~cm}$ for transcontinental distances (Robertson et al., 1979). This combination of precision and range should make VLBI a very powerful technique for monitoring the time dependence of regional and continental baselines. Yet it is still not widely used for this purpose. Why? A principal reason has been cost. Applications of VLBI to geo-

Proc. of the 9th GEOP Conference, An International Symposium on the Applicatioms of Geodesy to Geodsnamics, Ortoher 2-5. 1978. Dept. of Geodetic Science Rept. No. 280. The Ohio State Univ.. Columbus, Ohio 43210. desy have hitherto involved observations of the random, weak, radio signals received from distant, extragalactic, sources. The achievement of useful signalto-noise ratios with these sources has dictated the use of large diameter antennas, expensive atomic frequency standards, and wideband tape-recording and correlating systems.

In contrast, only very small, simple, and inexpensive ground equipment is required to utilize the relatively strong, precisely controlled, radio signals that can be transmitted from Earth satellites. Nonetheless, although several methods have employed satelite signals to determine baselines, none of these methods has yet achieved the measurement precision demonstrated with VLBI. Why not? What is the "secret ingredient" of the interferometric technique? Basically it is the use of differencing. Interferometry, per se, involves the differencing of the phases of signals received at the two ends of a baseline. With properly designed equipment, the inherent " $2 \pi$ " ambiguity in these phases of radio signals from a given source can be eliminated, and advantage taken of precise phase measurements of the signals from several sources, to determine a baseline with an uncertainty equal to a small fraction of the wavelength of the radio signals. Further, this baseline determination does not depend on the signals from any source having any particular temporal regularity.

The baseline vectors determined by radio interferometric techniques can be related to the best known approximation to an inertial frame: the positions in the sky of compact, extragalactic, radio sources. Of course, when the baseline vector is determined from interferometric observations of radio signals from satellites, an extra step is required to relate the positions of the satellites to those distant radio sources. Again, the technique used is interferometric but, here, use of the full panoply of the conventional VLBI armamentarium is required.

A system that combines the advantages of VLBI with the benefits of strong satellite signals could open a new era in geodesy. We describe a relatively simple system here. It would employ compact ground equipment with no moving parts and low-power radio transmitters on a set of satellites. We dub this combination the Mighty MITES system, MITES being an acronym for Miniature Interferometer Terminals for Earth Surveying (or, to be presumptuous and fācetious simultaneously. the Massachusetts Institute of Technology Engiñeering Success). Our system appears 
to be potentially more efficient than any other so far proposed for the three-dimensional monitoring of crustal strain accumulation and release over distances ranging from tens of meters to hundreds of kilometers. This system could also be applied to a variety of other surveying and navigation problems on land, sea, and air, and in space.

In the remainder of this paper, we describe a preliminary design of the proposed system, the operation of the system, the limits on the achievable accuracy, an inexpensive method to demonstrate the feasibility of the system, some of its possible applications, and, finally, a comparison of the system with other space geodetic methods.

\section{System Description}

In this section we discuss, in turn, the basic concepts underlying our proposed system, our preliminary thoughts on the design of the relevant satellite and ground equipment, and some possible design modifications.

\section{A. Basic Concepts}

Here we outline briefly some of the concepts upon which our proposed system is based.

1. Observable. The basic quantity that would be measured with this system is the interferometric phase -- the difference between the phases of radio signals from a single satelitite received at any two terminals. These phases would each be obtained simultaneously for a set of different radio frequencies ("tones") covering a wide, 1 octave, band so that (i) the effect of the ionosphere could be virtually eliminated, and (ii) the inherent " $2 \pi "$ ambiguity of the phase observable could be resolved. Measurements of the frequencies of the received signals would be made concurrently to enable conversion of the phase differences to corresponding delay differences.

2. Baseline Determination. These delay differences could be interpreted in a standard manner to determine the components of the baseline vector. For observations of a given satellite at a given instant, the interferometric phase delay can be expressed approximately as

$$
\tau \simeq \frac{1}{\mathrm{C}} \overrightarrow{\mathrm{B}} \cdot \hat{\mathrm{s}}+\tau^{\mathrm{cl}},
$$

where $\hat{\mathbf{B}}$ is the baseline vector, $\hat{\mathrm{s}}$ is a unit vector in the direction of the satellite being observed, and $\tau$ cl represents the difference between the epoch settings of the clocks at the two terminals (i.e., their departure from synchronism). For the purpose of this simplified explanation, we assume $B \ll r_{S}$, where $r_{S}$ is the minimum distance of a satellite from a terminal, and we suppress the effect of the difference in rate of the clocks at the two terminals (see section III). Equation (1) shows that at any instant, the observed delay contains information on the projection of the baseline vector along the direction to the satellite, and on the clock-synchronization error. To determine all three components of the baseline vector simultaneously with the synchronization error at any instant, the signals from at least four satellites must be observed and the resultant four linear equations solved for the four unknowns. For a unique solution, the satellites cannot all appear to lie on the same circle in the sky. If they do, then the component of $\vec{B}$ in the direction of the center of this circle cannot be separated from $\tau^{\mathrm{Cl}}$. (See also section III.A.)

3. Reference system. We assume that the positions of the four (or more) satellites observed are known with respect to a well-defined coordinate system. Different coordinate systems could be utilized, depending on the application. We consider one example (see, also, Section V): a nearly inertial coordinate system with an orientation defined by the directions of extragalactic radio sources. Such a system could be used if three (or more) base stations made continual differential interferometric phase observations of the sateliites with respect to those extragalactic radio sources that appear in the same part of the sky (Counselman et al., 1972; Preston et al.,1972). Given that the vector positions of the base stations with respect to the directions of the extragalactic sources were already known, virtually instantaneous measurements of the differential interferometric phases between a satellite and an extragalactic source from observations from each of two independent baselines suffice to determine the satellite's direction in this nearly inertial frame. Thus, the vector baselines between our compact ground terminals could be related to this frame through their coordinates in the satellite frame. The base stations would of course require conventional VLBI instrumentation to observe the extragalactic radio sources. As long as the direction of the comparison extragalactic source is neither too far from that of the satelite nor too near that of the sun, the observations of the satellite need be made only of the highest transmitted tone.

We defer until section IV discussion of the limits on achievable accuracy in the determination of the baseline vector with respect to a reference frame. 
4. Global Positioning System. The satellites of the NAVSTAR Global Positioning System (GPS) (Parkinson, 1976), scheduled for launch during the next few years, will have orbits almost perfectly suited to our proposed VLBI system. In the planned steady-state configuration, there will be 24 such satellites, eight distributed in each of three orbital planes, spaced by $120^{\circ}$ in nodal longitude. Within each plane the satellites will be approximately evenly distributed around a nearly circular orbit of $\sim 63^{\circ}$ inclination, $\sim 20,000-\mathrm{km}$ altitude, and $\sim 12$-hour period. As a result, at any place on the surface of the Earth at least six satellites will always be visible, and of these at least four will be suitably distributed (i.e., not all on or near any single circle in the sky):

The expected useful lifetime of a GPS satellite is five years with a maximum of about seven set by the amount of stored gas for the attitude-control system. Thus, in principle at least, there will be continual opportunities to modify the payload to accommodate our proposed system.

\section{B. Satellite Equipment}

We now describe the equipment we propose to place on each satellite. First, we discuss the factors governing the choice of subsystems and then each of the subsystems. For definiteness, we consider the design in the context of the GPS satellites.

1. Requirements. What special requirements must be satisfied by the equipment aboard the satellites for our proposed system to be operated successfully? First of all, the satellite must transmit a sufficiently strong and stable signal. The signal must also be structured to allow the user to resolve the "2 $2 \pi$ " ambiguity in the interferometric phase. In effect, the same cycle of the signal must be identified at both ends of the baseline. The usual method of making such an identification in VLBI is to combine observations made simultaneously at several different frequencies, or tones, $f_{i}(i=1$, $2, \ldots)$. The respective ambiguity spacings in delay, equal to $1 / f_{j}$, are different, and the overall delay ambiguity can be resolved if, within the range of possible delays allowed by a priori information, there is a unique delay that is consistent with the phase observations at all frequencies. The task of resolving the phase ambiguity is complicated, however, by the dispersion of the propagation medium and by possible interference from reflected signals (see Section VII). The set of frequencies used must be chosen with these complications in mind.

Since, for the range of frequencies of possible interest, the Earth's ionosphere introduces a delay proportional to the inverse square of the frequency of the signal, it would appear desirable to employ the highest possible frequencies with the proposed system. However, we must also consider our paramount desire for a simple system. This desire to avoid, for example, high-gain antennas and high transmitted power levels places an upper bound on the usable frequency. Since, for given antenna directivities and transmitted power, the received power is inversely proportional to the square of the frequency, sufficiently high signal-tonoise ratios can only be obtained simply at low frequencies. The optimum range of frequencies is approximately $1-2 \mathrm{GHz}$, $1 \mathrm{i}-$ mited on the low end by the ionosphere and on the high end by signal-to-noise ratio considerations. An approximately one octave spread in the frequencies of the tones is important for the elimination of $2 \pi$ ambiguities in the face of both the ionosphere and the possible interference from reflected signals.

Within this one-octave range of frequencies there are several wide bands allocated under existing international regulations for purposes that would encompass those of the proposed system (International Telecommunications Union, 1968). This system requires relatively little spectrum space -- no more than ten narrow bands, each at most $100-200 \mathrm{kHz}$ wide, within the $\sim 1-t o-2 \mathrm{GHz}$ range. The precise placement of these bands within this range is quite flexible and could be adapted to existing constraints. Basically, we require one band near the low-frequency limit, one near the high-frequency limit, and the others distributed between these limits. The inter-band spacings would range from a minimum of $\sim \mathrm{MHz}$ to a maximum of $\sim 400 \mathrm{MHz}$. The spacings could be in approximately geometric progression but do not have to vary monotonically with frequency (see Section III for further discussion).

2. Transmitter. To satisfy these requirements, each satellite could be equipped to transmit an unmodulated circularly polarized continuous wave (CW) with $\sim 1$ watt of power in each of up to ten bands. To avoid having to contend with interference between signals received from different satellites, each of these bands could be subdivided into contiguous, nonoverlapping "channels". Since each GPS satellite could share a channel with that other satellite spaced 180 deg apart in longitude, the number of channels need only be 12. Setting the width of each channel equal to $8 \times 10^{-6}$ of its center frequency would allow for a tolerance on transmitted frequency of \pm 1 part in $10^{6}$, as well as for the extremes of Doppler shift, of \pm 3 parts in $10^{6}$, that could be 
observed on Earth for signals from satellites in orbits of the GPS type. Thus, the total. width of the band of 12

channels at $\sim 1 \mathrm{GHz}$ would be $\lesssim 100 \mathrm{kHz}$, and at $\sim 2 \mathrm{GHz}$ would be $\lesssim 200 \mathrm{kHz}$. Should less of the spectrum be available, modifications are possible to compensate, as discussed in Section II.D. Here we proceed on the assumption that no modifications will be required.

Transmitters are avaijable commercially which yield 1 w of radio-frequency output for $3.6 \mathrm{w}$ of d.c. input power. Such devices have dimensions and mass of about $5 \mathrm{~cm} \times 5 \mathrm{~cm} \times 10 \mathrm{~cm}$ and $0.9 \mathrm{~kg}$, respectively. Thus, for a total of ten bands, $36 \mathrm{w}$ of DC power and a volume of 0.0025 $\mathrm{m}^{3}$ would be required. These transmitters have an adequate long-term frequency stability of 1 part in $10^{6}$ and a more than adequate spectral purity of $\sim 1$ part in $10^{10}$ : The overall volume and mass could undoubtedly be lowered for use in space, especially if ten transmitters were packaged together. The total DC power requirements could also be lowered by some combination of an insignificant sacrifice in spectral purity and a use of a single oscillator to generate multiple tones through modulation which, itself, requires little power. However, to maintain high reliability, one would prefer not to eliminate too much redundancy.

3. Power Combiner. The outputs of the separate transmitters would be combined before being fed to an antenna. Design of such a combiner would be straightforward, involving mainly a set of resonant, low-loss circuits.

4. Antenna. We envision each satellite equipped with a circularly-polarized antenna of modest directivity $\left(30^{\circ} \lesssim\right.$ beamwidth $\lesssim 60^{\circ}$ ), which illuminates the entire visible portion of the Earth approximately uniformly. Such an antenna would have at least $10 \mathrm{db}$ gain, but would be physically small and would not require precise pointing. A suitable antenna would also have a bandwidth sufficiently broad to yield this gain over the span from 1 to 2 GHz. (Variations in the transmitting antenna's gain, as well as in the receiving antenna's effective aperture, at the frequencies of the various tone transmissions, could be compensated by suitable adjustments in the relative transmitted powers.) Types of antennas that might satisfy these requirements include cavity-backed spirals and conical spirals. The sizes and masses of such antennas are quite modest. For example, a commercially available cavity-backed spiral for these frequencies has a gain of nearly $10 \mathrm{db}$ and is about $12 \mathrm{~cm}$ in diameter and $10 \mathrm{~cm}$ deep, with a mass of about $1 \mathrm{~kg}$.
These characteristics of the satellite equipment are summarized in Table 1 and an overall block design is shown in Figure 1. This equipment would, of course, require integration into the GPS configuration. Questions of location, mass, volume, and heat generation and dissipation all must be addressed. The only positional requirement is that the antenna must face earthward; the other major requirement is, of course, for the appropriate supply of direct current. Any requirement for uplink and downlink telemetry would be very modest, because the system is so simple.

\section{c. Ground Terminal}

The most important feature of the transmitter described in section II.A is that it enables a relatively simple ground terminal to be used. Each such ground terminal would consist of an antenna, a receiver, a frequency and time standard, digital counting and timing circuitry, a calibration signal generator, atmospheric sensors, control logic, a system to store and/or telemeter data, and a power supply. We discuss these components, each in turn, after first describing some of the requirements that the ground terminal must meet.

1. Requirements. The ground terminal must have an antenna matched in circular polarization to the satellite antenna, but with little directivity. We wish to avoid the complexity and expense of re-pointing the receiving antenna, by either mechanical or electrical means. Further, the antenna must receive signals simultaneously from widely separated directions in the sky, with elevation angles as low as $10^{\circ}$. The receiving antenna must also operate over an approximately 2-to-1 range of frequency, corresponding to a wavelength range of approximately 15 to $30 \mathrm{~cm}$.

With any receiving-antenna design, it is also necessary to ensure that the phase of the signal received directly from a satellite is not altered significantly (see Section VII) and in an unknown way through interference that could arise from waves reflected or scattered to the antenna from the ground. The significance of such phase errors depends, of course, on the desired geodetic accuracy. In Section IV.C, we show that the errors in baseline determination would generally be comparable to the equivalent path-length errors in the measurements of the interferometric phase delays. Thus, for example, to achieve one centimeter baseline accuracy, would require phase errors under $24 \mathrm{deg}$ for $f \approx 2 \mathrm{GHz}$. Equivalently, the reflected signal would have to be at least $7 \mathrm{db}$ weaker than the di- 
rectly received signal. For one millimeter geodetic accuracy, the field strength of the reflected and scattered signals, and/or the antenna's sensitivity to such signals, would have to be attenuated tenfold (20 db in power) further. On the other hand, if geodetic precision, rather than accuracy, were the goal, as in some applications, one could tolerate a more cluttered and uneven environment for the receiving antenna because the distribution of azimuths and elevations of the GPS satellites as viewed from any given location repeats approximately with a period of 12 hours, so that the signal-: reflection pattern and the consequent electrical phase variations would also be repeated periodically.

2. Antenna. The requirement that the phase of the signals received directly from the satellite not be altered significantly by signals reflected or scattered into the antenna could be satisfied by ensuring that the receiving antenna has an unobstructed view of the sky above $10^{\circ}$ elevation, and that no large, elevated planar reflectors such as building walls or fences are situated so as to reflect satellite signals toward the antenna. The antenna also would have to be placed above a $\sim 30$ to $90 \mathrm{~cm}$ diameter metal "ground screen" of sheet metal or stiff wire mesh placed flush with the ground. The exact diameter needed and the restrictions on the local terrain would depend on the desired accuracy. The screen would also serve to reduce pickup of thermal "noise" radiated from the ground. (This method of siting an antenna is commonly used for high-accuracy measurements of microwave antenna radiation patterns).

The antenna would be mounted in the center of the ground screen and could have various forms. We describe one possible antenna design here. Mechanically somewhat complicated, but with apparently more than adequate electrical properties, this antenna would consist of a simple stack of crossed pairs of horizontal, half-wavelength dipoles. They would be made of metal rod or tubing with each pair cut for one of the, at most, ten frequency bands. Of course, the closely-spaced bands could be served by a single pair of dipoles. Each dipole pair would be placed three-eighths of a wavelength above the ground screen. The two orthogonal dipoles comprising each pair would be fed in phase quadrature for circular polarization. Each pair could be connected to a separate, narrowband, receiver preamplifier if desired; more than one pair could also be connected efficiently to a single broadband preamplifier.

With a height of $3 \lambda / 8$ above a horizontal ground plane, the dipole pair has greater than unity gain relative to a circularly polarized "isotropic" antenna at all elevation angles above about $20^{\circ}$, and its gain is about $-5 \mathrm{db}$ at $10^{\circ}$ elevation, although the response approaches zero at the horizon. There would also be negligible ohmic loss with this antenna.

A crossed-dipole stack would have to be specially designed and tuned to the MITES systein frequencies. However, this task is rather simple, and the cost of replicating the resultant antenna in moderately large quantity would probably be under $\$ 50$ per unit.

Finally, we note that the phase-shift characteristics of the antenna itself, in conjunction with its ground screen, would be unimportant if identical antennas were used for all terminals; but, in any event, the antennas could be calibrated on a test range.

3. Receiver. The receiver could have a simple, uncooled, transistor radio-frequency (RF) front-end amplifier, or possibly two or more such amplifiers, each tuned to a different portion of the 1-to-2 GHz range spanned by the transmitted signals. In either case the total volume and mass of the front end would be under $100 \mathrm{~cm}^{3}$ and $0.1 \mathrm{~kg}$, respectively. A system noise temperature of $200^{\circ} \mathrm{K}$ could be easily achieved and would be sufficiently low, given the characteristics of the transmitted signals and the antennas described above. No difficult gain or phase stability demands would need to be met, because the output of the final amplifying stage of the receiver would be hard-limited or "clipped", and because delays of the signals through the receiver would be separately monitored, as discussed in subsection 6 .

After passage through the front-end amplifier, the signals received in each of the 100-to-200-kHz-wide bands would be mixed with a fixed-frequency local oscillator(LO) signal, derived by coherent multiplication from the master oscillator, to convert them to an intermediate-frequency (IF) band centered at $\sim 200 \mathrm{kHz}$. The value of $200 \mathrm{kHz}$ was chosen to minimize the requirements on time resolution in the subsequent measurement of the phase of the IF signals (see Subsection 8 ). No lower value could be chosen because of the desire to keep the ratio of the frequencies of the highest and the lowest IF signals below three in order to prevent interference from the odd harmonics generated by clipping. The down conversion to IF might be accomplished in a single stage with a quadrature-phasing, single-sideband mixer of the type described by Rogers (1971), or depending on considerations of dynamic range, stability, etc., two or more conversion stages might be used. 
4. Time and Frequency Standard. Since observations would be made of at least four satellites simultaneously, the departure of the clock at each terminal from synchronism in epoch (and rate) with the clock at every other terminal can be determined from the observations as explained in section II.A.2. A high order of stability is therefore not required of the time and frequency standard at each terminal. The primary requirement is for sufficient short-term frequency stability, of $\sim 1$ part in $10^{10}$, to maintain phase coherence during each, approximately one second, period of coherent integration. Long-term stability of $\sim 1$ part in $10^{6}$ is desired to facilitate acquisition of the satellite signals. The need for the $\sim 1-$ sec observation periods of different terminals to overlap substantially implies that time must be kept at each terminal to an accuracy better than about 0.2 seconds, unless the observations are made continuously. In the latter case, interpolation between successive observations could be used to "match" time tags. Otherwise, drift in epoch error would have to be monitored and the clock in each terminal would have to be reset periodically, via telemetry if necessary. (See, also, Section III.A.)

These requirements on frequency and time are readily met by a compact, commercially-available, crystal oscillator. We shall henceforth refer to this as the "master" oscillator.

5. Digital Counting and Timing Circuitry. The digital counting and timing circuitry comprise the heart of the terminal and we describe them in some detail here. For convenience in description, assume that the received signals have been converted to the IF band, centered at $\sim 200$ $\mathrm{kHz}$, as mentioned in Subsection 3. Each of these IF signals, one for each of the up to ten RF bands, would be bandpass Filtered to approximately 100 to $300 \mathrm{kHz}$ and symmetrically limited or "clipped" as mentioned earlier, to obtain a two-level signal which would be switched via "logic" circuits to selected inputs of a set of approximately 20 identical modules (see Subsection 8 ). Each such signal could be directable to the input of any module, according to the plan discussed below, although a more efficient arrangement is possible. The function of each module would be to measure the phase and the frequency of one signal from one satellite.

The IF input to a module would be fed to a second-order phase-locked loop whose bandwidth under the expected conditions of $+10 \mathrm{db}$ or greater signal-to-noise ratio (SNR) could be switched to values of either $7.5 \mathrm{~Hz}$ or $30 \mathrm{~Hz}$, and whose tracking range in either case would span the IF band of from 100 to $300 \mathrm{kHz}$. (Note that the SNR is inversely proportional to the bandwidth of the loop and that the power received by the crossed-dipole antenna will be inversely proportional to the square of the radio frequency. Hence, under the assumption that the effective radiated power from each satellite is the same for all tones, the loop bandwidth for reception of the $2-\mathrm{GHz}$ signals must be fourfold smaller than the loop bandwidth for the $1-\mathrm{GHz}$ signals in order to maintain the same value of SNR.) The loop would be locked to, and would track, the first suitable satellite signals encountered in this band. The loop SNR would be at least $+10 \mathrm{db}$, and the corresponding root-mean-square (rms) random phase error in the loop output would be less than $13^{\circ}$, given (i) the effective transmitted power of $\sim 10 \mathrm{w}$ ( $1 \mathrm{w}$ transmitted, coupled with $10 \mathrm{db}$ of antenna gain); (ii) the worst-case satellite elevation angle of $10^{\circ} ;$ (iii) the crosseddipole receiving antenna; (iv) the receiving system noise temperature of $200^{\circ} \mathrm{K}$; (v) the 2-db clipping loss; and (vi) the $30-\mathrm{Hz}$ and $7.5-\mathrm{Hz}$ loop bandwidths for the 1-GHz and 2-GHz signals, respectively. The dynamic tracking error due to the time-rate-of-change of the input signal frequency would be well under $1^{\circ}$, as would the static phase error, even with the use of the narrower loop bandwidth for the highest-frequency signals. The loop output would be fed to digital circuits which would perform two functions: (i) a continuously accumulating count of the integral cycles of the locked oscillator; and (ii) an accurate measurement, modulo one cycle, of the oscillator phase relative to that of the receiver clock. The instantaneous value of the accumulated cycle-count would be sampled nondestructively at selected times, according to the clock. The phase measurement would also be made at selected times, with $\sim 100$ ns time resolution, equivalent to $\sim 0.02$ cycle phase resolution at the $\sim 200 \mathrm{kHz}$ IF. This measurement could be made with a digital start $\div$ stop counter with a "clock" rate of $10 \mathrm{MHz}$. The count would be initiated by a command from the ground terminal clock, and would be halted appropriately by the output of the locked oscillator, for example, by the next occurring positive-going transition. Averaging would be performed by timing a succession of such time intervals (i.e., by restarting and restopping the count) over a time span of approximately one second.' By virtue of this averaging, the rms random noise of the equivalent phase measurement could be reduced to less than $5^{\circ}$, the equivalent of $2 \mathrm{~mm}$ of electrical path length at a frequency of $2 \mathrm{GHz}$. (The equivalent noise bandwidth of the measurement would be $\therefore 2 \mathrm{IIz}$ instead of $\sim 1$ $\mathrm{Hz}$ because of the image "fold over": for 
the 2-GHz signals, the SNR in this bandwidth would be >18 $\mathrm{db}$.)

The phase-locked-loop portion of each module could be implemented with lowpower, analog, integrated circuits and low-power Schottky transistor-transistor logic (TTL) circuits. All of this circuitry combined would consume less than $0.5 \mathrm{w}$. Continuous cycle-counting could be performed by a standard, low-power, "large-scale integrated" (LSI) circuit that also would consume about $0.5 \mathrm{w}$ of power. The 100-ns resolution timing could be implemented with standard TTL circuits that would use approximately 2 w. However, power would have to be supplied to the latter circuitry only for the $\sim$ l second interval during which the fractional-cycle phase measurement was being made. For many applications, these measurements might be repeated infrequently, at several-minute intervals, for example. In such cases, the average power consumed by each module would remain at the $\sim 1 \mathrm{w}$ level required to sustain the phase-locked-loop and cycle-accumulating circuits. Inasmuch as the complete receiver would require about 20 such modules, low power consumption per module is an important advantage.

The feasibility of building a module with the characteristics described has been demonstrated by the differential Doppler receiver developed by $H$. F. Hinteregger and one. of us (C.C.C.) and applied to differenced VLBI observations of the signals transmitted from the Apollo lunar-surface experiments packages (Counselman and Hinteregger, 1973; Counselman et al., 1972; see also Figure 3 in Counselman, 1976 ).

6. Calibration Signal Generator. Signals from satellites would undergo delays in the RF amplifier(s), the mixers, and the IF amplifiers of the receiver. These delays could be calibrated by injecting a suitable signal of low power directly into the $R F$ input of the receiver. The calibration signal could consist of a periodic train of pulses, each $\leqslant 20$ ps in duration and all derived directly from the master crystal oscillator. A pulse repetition frequency of $\leqslant 100 \mathrm{kHz}$ would be chosen so that at least two of the harmonics of this calibration signal would appear within the passband of each IF amplifier. Two harmonics are required to monitor the group delay as well as the phase delay variations of the receiver. The group delay -- the variation of phase with frequency -- is important to monitor because the signals received from different satellites have different frequencies.

The phases of the calibration-signal harmonics would be measured in exactly the same way as would the phases of the satellite signals. This method has been used successfully in the calibration of other geodetic VIBI receiver systems, at the millimeter level of accuracy (Whitney et al., 1976; Rogers et al., 1978). When combined with the results from just one set of measurements of the phase-shiftvs.-frequency characteristics of the IF portions of the receiver, carried out when these are originally built, the information from the calibration signals should serve to account for all receiver phase variations to within an uncertainty of under 1 deg. The calibration signals would not have to be monitored continuously, but could be checked, for example, before and after each satellite tracking period as discussed below.

7. Atmospheric Sensors. The delay of the radio signals introduced by the neutral atmosphere must be modeled accurate$1 y$, as discussed in section IV.A. Thus we would include a suitably compact, electronically-readable barometer, thermometer, and hygrometer in each interferometer terminal. Such instruments are available commercially and are incorporated, for example, in some compact satellite Doppler-tracking receivers. If the data from a particular set of terminals are to be analyzed at a central location, it may be feasible and more economical to omit the sensors from the terminals and to interpolate from weather-station records to obtain the values of the atmospheric parameters (see section IV.A).

8. Control Logic. All of the control functions to be carried out in the ground terminal could be automated straightforwardly through the use of an integratedcircuit microprocessor. Routine functions would include collection of data including the atmospheric information, the cycle-counts, the time-increments, and the received signal and noise levels. Less routine functions would include the determination of the proper phase calibration of the signais received in the different bands. The switching of the phaselocked loops to the appropriate frequencies for acquisition of the satellite signals would also be automated. For signal acquisition, at least two alternative strategies could be followed, each based on the controller's ability to (i) monitor the frequency of the oscillator in each phase-locked loop through cyclecounting; (ii) slew each such oscillator upward or downward in frequency; and (iii) note the amplitude of the coherently-detected signal from each phase detector.

If the controller were in two-way communication with a central processing station, this station could instruct the controller regarding the times and frequencies at which new satellites could be acquired. But it would also be possible, 
and often preferable, for the controller to operate automatically. At preset intervals, each of the 12 frequency channels in the lowest-frequency $(\sim 1 \mathrm{GHz})$ band would be swept by a phase-locked loop searching for a signal. (Note that it is vastly more efficient to conduct the search at the lowest frequency because (i) the loop bandwidth is wider; (ii) the loop time constant is shorter; and (iii) the spectrum to be searched is smaller. Since the first two factors each vary with the square and the third with the first power of the radio frequency, we conclude that the search time depends' on the fifth power of the radio frequency!) For the signal strengths and bandwidths given previously, approximately 10 seconds would be required for one loop to search completely one of these 8-kHz-wide channels. Of course, different loops would be programmed to search different channels simultaneously. Once a new sateliite had thus been acquired, others of the $\sim 20$ loops could be assigned by the controller to lock onto the signals transmitted in the other bands by the same satellite. Less searching would be required in this step, since the Doppler shifts would be known.

Whenever a satellite is acquired, the phases of all of the sinusoidal signals that it transmits would have to be measured simultaneously at least once, to later enable the interferometric phase ambiguities of the signals to be properly resolved. This task would require the simultaneous commitment of up to ten phase-locked loops to that one satellite. However, once this task, which would take only a few seconds, had been accomplished, only two loops would be needed to track the highest and lowest frequency signals from the satellite. Thus, after elimination of the $2 \pi$ ambiguities, it would be necessary to maintain a continuous count of the cycles received from these two signals only to remove ionospheric effects. In the steady state, simultaneous tracking of, say, six satelites would require twelve loops, and eight loops would remain for other tasks. These tasks would include the periodic monitoring of the phases and amplitudes of the calibration signals in all of the IF bands, and the searching for new satellites. To reach the steady-state condition after five of the six satelites have already been acquired will require up to 20 of the phase-locked loops -ten for the steady state tracking of the first five satellites and up to ten loops to enable the proper phase connection to be made for the signals from the sixth satellite. The total time to reach a steady state would, on average, be about one minute.

The control logic must also contain means to recognize any lock on a spurious signal and to reject this signal; in such a scheme, advantage must be taken of the known relation between the frequencies of the various tones transmitted by each satellite.

9. Data Storage and Telemetry: We estimate that about 3500 bits of data would need to be stored at, or transmitted by, each terminal for the initial determination of a baseline. This total includes allowances of 33 bits for the integercycle count, 9 bits for the fractionalcycle phase measurement, 12 bits for the measured value of frequency, and at least 1 error-flag bit to indicate loss of lock for each of the up to 10 signals received from each of 6 satellites, plus a few hundred bits for time tags and other labels, and for data on the atmospheric parameters. This quantity of data could be transmitted over an ordinary telephone line in 2-3 seconds. After the initial signal-acquisition and phaseambiguity-resolution operations had been completed, and when only two phase-locked loops were tracking each satellite, a baseline redetermination could be performed with an additional 1 kilobit of data from each terminal. Data from a large number of observations could also be stored within the terminal in inexpensive, solid-state memory devices, and could be collected later, for example by "dial-up" telephone. With the addition to the terminal of a l-watt microwave transmitter, the data could be relayed from a remote to a central location through a satellite repeater. Alternatively, if a telephone line were availa-

TABLE 1. Possible Characteristics of Satelite Equipment for Use with the Mighty MITES System

Equipment

Description/ Characteristics

TRANSMITTER

Frequencies

Polarization

Power

Total DC Input

Total volume

Total Mass

ANTENNA

Type

Bandwidth

Beamwidth

Gain

Dimensions

Mass $\leqslant 10$ tones spaced between 1 and $2 \mathrm{GHz}$ circular

$\sim 1 \mathrm{w}$ per tone

$<35 \mathrm{~W}$

$\sim 0.0025 \mathrm{~m}^{3}$

$\leqslant 10 \mathrm{~kg}$

cavity-backed spiral

$\sim 1$ to $2 \mathrm{GHz}$

-30 to $60 \mathrm{deg}$

$\leqslant 10 \mathrm{db}$

$12 \mathrm{~cm}$ dia $\times 10 \mathrm{~cm}$

deep

$\sim 1 \mathrm{~kg}$ 


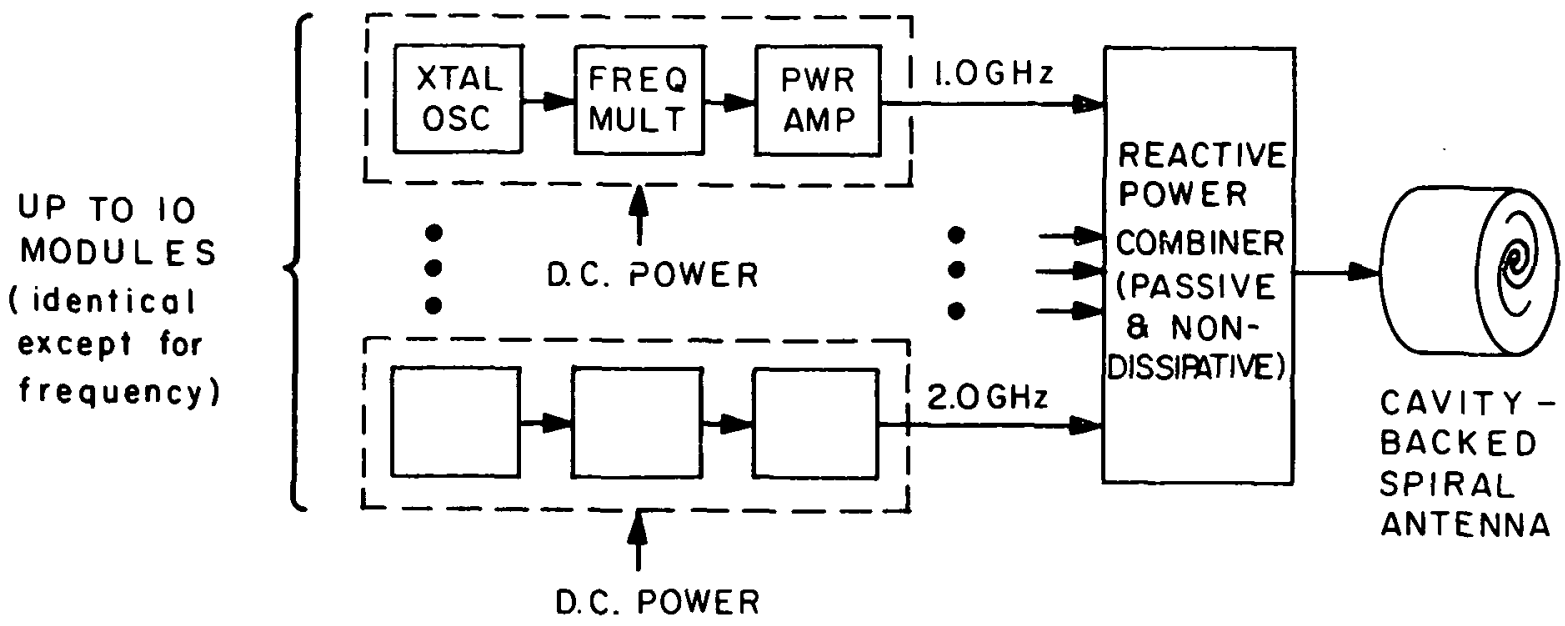

Fig. 1. Block diagram of radio transmitting equipment to be placed on satellite, for use by Mighty MITES.

ble within $\sim 50 \mathrm{~km}$, a UHF or VHF radio link and a telephone "patch" could be used.

10. Power Supply. The complete terminal as described would require about 50 watts of power. Thus, it could not operate continuously and unattended for long periods without a steady power source. For applications in which observations every second were not required, the average power consumption could be kept to about 1 watt, since most of the electronics, save the master crystal oscillator and the clock, would not have to be powered except during observations. In these applications, power might be drawn from batteries charged by an array of solar cells.

These characteristics of the proposed interferometer ground terminal are summarized in Table 2. See also Figure 2 .

\section{Possible Design Modifications}

The particular design we described was motivated mostly by the desire for simplicity and reliability. However, many modifications of the design of the Mighty MITES system could be made to accommodate different requirements or constraints. For example, if necessary, the bandwidth required for the system could be reduced substantially with perhaps negligible degradation of performance. It is likely that a simple analysis, yet to be performed, would show that satellites separated by only $90^{\circ}$ in orbital longitude could be assigned the same nominal transmitter frequencies without risk of mutual interference, because of the difference between the Doppler shifts of their signals as received on the Earth. If so, the number of channels, and the total bandwidth, could be halved. It might

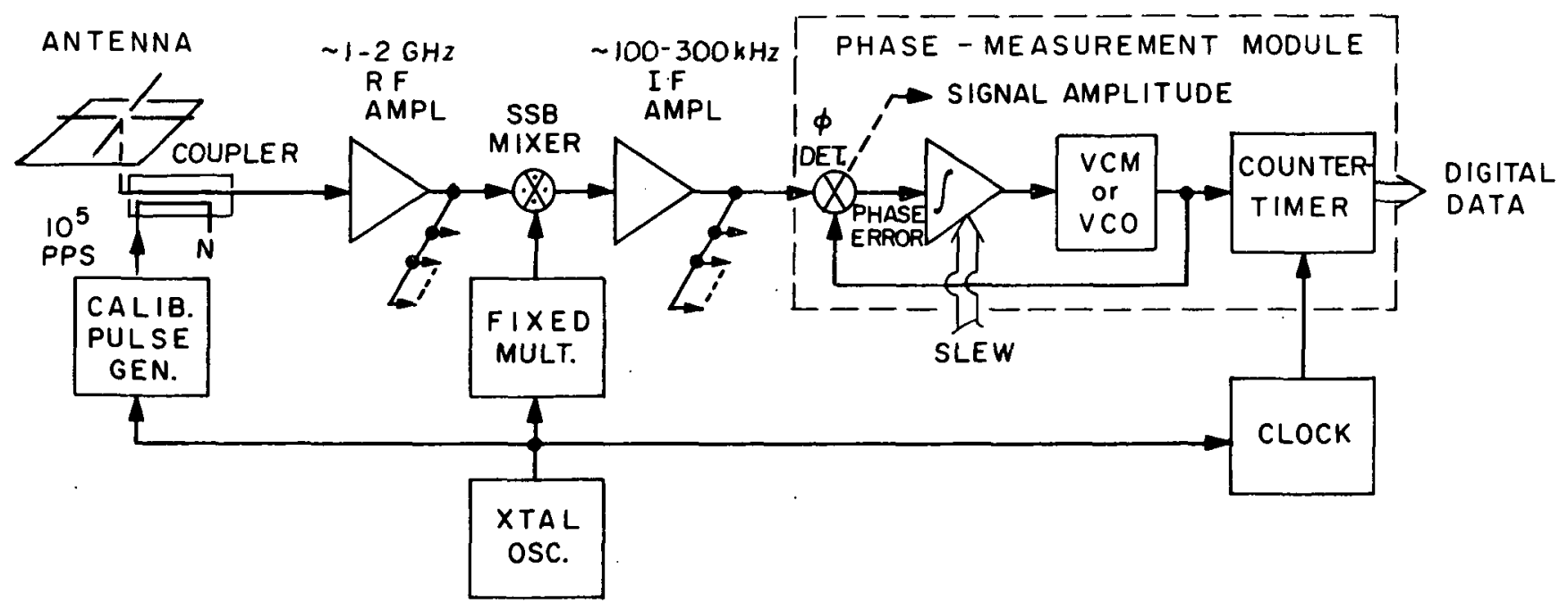

Fig. 2. Block diagram of radio signal receiving and processing portions of Mighty MITES ground terminal. One RF-to-IF converter and one IF amplifier are required for each of the up to ten RF bands. A total of 20 phase-measurement modules are required, as explained in the text. 
even be feasible to reduce the number of channels in each band to one; that is, all 24 satellites might have the same nominal transmitter frequencies. Typically, about eight satellites are simultaneously visible at a given ground location,

TABLE 2. Possible Characteristics of Ground Equipment for the Mighty MITES System

$\begin{array}{cc}\text { Equipment } & \text { Description/ } \\ \text { Characteristics }\end{array}$

\section{ANTENNA}

Type

Polarization

Frequencies

Gain

RECEIVER SYSTEM

Pre-amplifier (s)

Frequency Range

IF Amplifiers Center Frequency Bandwidth

Time and Frequency Standard

Short-term stability

Long-term Stability

Digital Counting and

Timing Circuitry

phase-Locked Loop Bandwidths

Time Resolution

RMS Noise in Phase

Measurement

Calibration Signal

Generator

Pulse Duration

Pulse Repetition

Frequency

Atmospheric Sensors

Controller
Crossed dipoles stacked $3 \lambda / 8$ above $\sim 30$ to $90 \mathrm{~cm}$ diameter ground screen

Selected coverage from 1 to $2 \mathrm{GHz}$ to match transmitted tones $>1$ relative to circular "isotropic" antenna for elevation angles $\geqslant 20$ deg: $-5 \mathrm{db}$ at elevation angle of 10 deg

Uncooled transistor (s)

1 to $2 \mathrm{GHz}$

$\leqslant 10$ units

$200 \mathrm{kHz}$

$200 \mathrm{kHz}$

Crystal oscilIator

$\sim 1$ part in $10^{10}$

$\sim 1$ part in $10^{6}$

$\sim 20$ units

7.5 and $30 \mathrm{~Hz}$

$100 \mathrm{nsec}$

$<5^{\circ}$ for one second integration in worst case

\section{$\lesssim 20$ psec \\ $\leq 100 \mathrm{kHz}$}

Barometer, thermometer, hygrometer

Integrated circuit microprocessor
Circular
TABLE 2, (continued)

Equipment

Description/ Characteristics

Receiver system (continued)

Data Storage or Telemetry

Capacity

1000-3500 bits per baseline determination

Medium Solid-state memory, cassette recorder, radio link and/or telephone line

Total Power

Peak

Average

$\sim 50 \mathrm{w}$

Total Mass

$\sim 1 \mathrm{w}$

Total Volume

$\sim 15 \mathrm{~kg}$

$\sim 0.1 \mathrm{~m}^{3}$

but the Doppler-shift and random, transmitter-frequency, differences would cause the eight received frequencies to be scattered throughout the width of the band. Two received signals would have frequencies differing by less than, say, $5 \mathrm{~Hz}$ probably less than 58 of the time. It should be possible to design a receiver to make accurate phase measurements on unmodulated signals which have similar strengths and frequencies that differ by as little as $5 \mathrm{~Hz}$; the receiver could also give an indication of when valid measurements were not obtained due to insufficient frequency separation between two signals. However, this approach might introduce considerable complication into the receiver. A more efficient approach, if it were desired to have many or all satelites sharing a single channel, might be to modulate the signal transmitted by each satellite with a unique, but narrowband $(\leqslant 1 \mathrm{kHz})$ "code" signal which could be detected in the receiver and used to discriminate between the transmissions from the different satellites. The latter approach is in fact used with the standard GPS navigational signals, except that the codes employed are rather complex and the modulation bandwidth is over $1 \mathrm{MHz}$. (The high GPS code complexity and modulation bandwidth are required for other purposes that are not essential to our system.) 


\section{System Operation and Costs}

In this section, we recapitulate briefly the operation of the Mighty MITES system with some more detailed emphasis on the technique for elimination of the $2 \pi$ ambiguity in the phase-delay measurements. We also comment very briefly on the costs of the system.

\section{A. Operation}

Each satellite would transmit a set of up to ten different tones. Each tone from each satellite would lie in a given radio-frequency ( $R F$ ) band. These bands, up to ten in all with each 100 to $200 \mathrm{kHz}$ wide, would be distributed suitably between $\sim 1 \mathrm{GHz}$ and $2 \mathrm{GHz}$. The ground terminal antenna, being approximately omnidirectional for elevation angles above 10 deg and sensitive to signals in each of these bands, would pick up the tones from those satellites within view. The incoming signals in each band would then be amplified and converted to an intermediate frequency (IF) band, centered at $\sim 200 \mathrm{kHz}$, by mixing the amplified signals with a local oscillator signal derived by coherent multiplication from the master crystal oscillator (Section II.C.4). The resultant IF signals would then be bandpass filtered, to select signals within a 100 to $300 \mathrm{kHz}$ band, and clipped to obtain a two-level signal that could be handled easily by digital circuitry. A subset of the $\sim 20$ modules (Section II.C. 8) that contain: phase-locked loops would be automatically directed to search for signals originating in the 12 different channels in the lowest $R F$ band (section II.B.2), since the efficiency of the search would be proportional to the inverse fifth power of the RF. (While the terminal is tracking signals from a given set of channels in this band, further search would be confined to the remaining channels.) After acquisition of a signal in the lowest band, the terminal would seek to acquire the signals in each of the corresponding channels in the up to nine higher-frequency bands. The known separations between the frequencies in the various bands would enable this search to be conducted very efficiently. Should the search fail in a sufficiently large number of bands, the originallyacquired signal would be assumed to be spurious and a new search of the channel in the lowest RF band would be undertaken picking up from the frequency of the spurious signal. Only ten seconds would be required for a module to search completely each $\sim 8 \mathrm{kHz}$ wide channel (Section II.B. 2) in the lowest $\mathrm{RF}$ band as the loop bandwidth would be $\sim 30 \mathrm{~Hz}$ (Section II.C.5), and its time constant $\sim 0.03 \mathrm{~s}$. Any module would also be able to track any signal in the total $200 \mathrm{kHz}$-wide IF band. The signal-to-noise ratio for the satellite signals received would be sufficient for these operations to be carried out for elevation angles as low as $\sim 10$ deg.

The outputs from the set of loops which had been locked to the up to ten tones transmitted by a satellite, would be analyzed by digital circuits to (i) accumulate a continuous count of the number of integral cycles made by the locked oscillator in the loop; and (ii) measure, modulo one cycle, the phase of this signal, relative to the clock in the terminal which is controlled by the master crystal oscillator. These measurements, combined, would constitute the basic one to be made by each ground terminal: the total phase of each of the up to ten tones received from each of the satellites being tracked. These phases would also be calibrated for the effects of delays within the receiver (Section II.C. 6). The resultant total phases would be suitably averaged, say over one second, and time tagged according to the clock in the terminal. After initial acquisition, it might be sufficient for some applications to continue to monitor only the phases of the signals in the highest and lowest frequency channels for each satellite (see Section II.C. 8).

In one mode of operation, these averaged data would be transmitted, along with auxiliary information including the measured values of the frequencies of the signals (Section II.C.9), from each terminal to some central location. At this central location, the data from any pair of terminals could be analyzed to (i) remove the $2 \pi$ ambiguity and, simultaneously, the effect of the ionosphere; and (ii) estimate the components of the baseline vector between the locations of the two terminals. To perform this task, additional information is needed, namely the positions of the satellites as functions of time. Crude or refined, a priori, information on terminal locations could also be used.

The radio frequencies of the tones would be distributed between $\sim 1$ and $2 \mathrm{GHz}$ in a manner designed to facilitate removal of the $2 \pi$ ambiguities in the interferometric phase delays. This distribution would involve spacings in frequency that are nearly in geometric progression, starting from a minimum spacing of about I MHz. (A strictly geometric progression would not be used because (i) a priori knowledge, e.g. of the ionosphere, would be incorporated in the choice of the first few spacings; (ii) the total spread would be limited by other constraints; and (iii) we would wish to be conservative.) 
The phases of each of the signals measured by each terminal could be converted to delay via use of the measured value of the radio frequency. The $2 \pi$ ambiguity and the ionospheric effects would be eliminated from the resulting set of up to ten interferometric phase delays at each measurement epoch by a "bootstrap" algorithm in which the ambiguity is eliminated first between the delays that result from measurements at the closely-spaced pairs of frequencies. These frequencies would be placed at the high, $2 \mathrm{GHz}$, end of the total band since the uncertainty in the ionospheric effects would be lowest there. Apart from the effects of noise, the shape of the ambiguity-free phase-delay vs. frequency curve is accurately known and, of course, taken into account in the algorithm. We omit the detailed presentation of this fairly straightforward algorithm to spare the reader the superficial complications engendered by symbols being festooned with the three sets of subscripts and superscripts necessary to distinguish the different satellites, ground terminals, and tones.

For continuing observations of the same satellites from the same terminals, the ambiguity and ionospheric elimination algorithms could be largely bypassed after the initial elimination. only the interferometric phase delays for the highest and lowest tones need be followed continuously; these could be combined very simply to remove the ionospheric effect. Similarly, for terminals closely-enough spaced for ionospheric effects to be negligible, the ambiguityremoval algorithm could be simplified somewhat.

The corrected interferometric phase delays for the satellites tracked from a pair of terminals would be analyzed by, say, a standard least-squares algorithm for each epoch to determine the vector baseline, as outlined in section II.A.2. Variations in clock behavior at either terminal over the signal integration interval would not affect the baseline result since each satellite is observed at the same times from a given terminal. The effect of such variations would therefore cancel because of the linearity of Equation (1), which would cover the same time interval for the observations of each satellite. However, a "commonmode" error in the epochs of the clocks at the two terminals would affect the baseline result because the assumed positions used for the satellites would be incorrect. Time tags on the incerferometric data accurate to a millisecond would reduce this error to a tolerable level. Such clock accuracy could easily be maintained for terminals in two-wày communication with a central processor. It would also be possible to obtain this information directly from the signals transmitted by the GPS satellites in their normal mode of operation. Alternatively, with observations of at least five satellites simultaneously, one could solve for this common-mode epoch error: In general, at least six satellites would be tracked simultaneously and thus some redundancy would be retained.

\section{B. Costs}

It is obviously premature to discuss in detail the costs of a Mighty MITES system. However, a few general statements can be made. The ground terminal, if replicated in reasonably large numbers, should not cost more than a few thousand dollars per unit which would make the capital equipment costs negligible compared to the overall costs of the system which would likely be dominated by labor costs. Because of their potential simplicity and reliability, and ability to be operated unattended, the Mighty MITES could, however, allow the labor costs to be reduced relative to those for many other monitoring systems (see section VII).

The cost of the equipment to be added onto the GPS satellites, if amortized over a large number of ground terminals, might not add significantly to the overall systems costs. Neither would the cost of a central computer be significant since most of the computations are carried out in the ground terminals. However, the development costs for both the ground-based and the satellite parts of the system, despite their intended simplicity, would likely be of the order of a million dollars.

\section{Limits on Attainable Accuracy}

The accuracy attainable in baseline determinations will be limited primarily by errors in knowledge of (i) the propagation medium and (ii) the positions of the satellites. The effects of both of these sources of error increase, albeit differently, as the length of the baseline increases; we discuss each in turn. Finally, we discuss the less important limits due to the relative geometric configuration of terminals and satellites, and due to instrumental effects.

\section{A. Propagation Medium}

The propagation medium contains, in effect, two components: the ionosphere and the atmosphere. The influence of the former can be virtually eliminated, as described in section III, by utilizing its dispersive nature. The tropospheric 
effect on the electrical path length of the radio signals is harder to determine because the neutral atmosphere is nearly non-dispersive throughout the radio band of frequencies. This effect, typically about $8 \mathrm{~ns}$ at the zenith, is variable by about 1 ns, due mainly to variations in the amount of water vapor in the atmosphere. If ground-level measurements of atmospheric pressure, temperature, and dew point are used to calculate the atmospheric zenith delay. the rms error in the result may be reduced to approximately 2 to $3 \mathrm{~cm}$ (Murray and Marini, 1976). However, for baselines of a few kilometers or less in length, and for sites at nearly equal elevations above sea level, the atmospheric delays introduced at the two ends of the baseline tend to cancel to a high degree. Our limited experience with such short baselines shows, for example, that tropospheric effects can be lowered to the millimeter level (Rogers et al., 1978) and, in appropriate climates, to the tenth millimeter level (Elsmore and Ryle, 1976) for baselines of length up to five kilometers. For long baselines, a series of interferometric measurements by our group (Robertson et al., 1979) involving the Haystack and Owens Valley antennas, separated by nearly $4,000 \mathrm{~km}$, demonstrated that a dozen baseline length determinations, distributed over a one and a half year period; show repeatability at the three centimeter level with the use of only surface measurements of atmospheric parameters. Higher accuracy results might be obtainable through use of water-vapor radiometers (see, for example, Schaper et al., 1970 and Moran and Penfield, 1976) to monitor the watervapor content above each terminal; perhaps the contribution of the troposphere to the uncertainty in baseline-vector determination could thereby be reduced to the centimeter level in all three components even for baselines of transcontinental dimensions. However, the efficacy of water-vapor radiometers for this purpose has yet to be established reliably in VLBI experiments under various climatic conditions. Moreover, at the present stage of technology, the water-vapor radiometers would be much larger, and more expensive, than our proposed ground terminals -- the use of such radiometers would be similar to the tail wagging the dog.

One can also take advantage of averaging. Since a baseline determination can be made, on average, once per second, one can afford for most applications to average the results for many minutes or longer. Further, with more than four satellites observed simultaneously, another form of redundancy is possible.
For both types, one can use the level of stability of the data and of the results as an indication of the accuracy of the baseline determination.

\section{B. Satellite Positions}

Errors in our knowledge of satellite positions are muted in their effects on baseline determination by the ratio of the baseline length to the satellite altitude. Thus for baseline lengths of a few hundred kilometers, the sensitivity to satellite position errors is reduced by a factor of nearly two hundred for satellites of the GPS type. For example, to achieve "instantaneous" accuracies of $2 \mathrm{~cm}$ over a $100-\mathrm{km}$ baseline would require satellite position errors smaller than $4 \mathrm{~m}$. (Note that for a baseline short compared to the satellite altitude, the interferometric delay is sensitive primarily to the direction of the satellite, not to its altitude). For transcontinental baselines, the immunity factor is under ten and satellite position errors become of correspondingly greater importance. But, as discussed earlier, effects of satellite position errors might be reducible to the level of the atmospheric errors if base stations were equipped to tie the satellite positions continuously over the required periods to those of extragalactic objects through differential VLBI observations. Averaging the baseline results over long periods of time, and, hence, over many satellites, would be acceptable for many applications and would tend to reduce the effects of satellite position errors.

\section{Geometry}

We now consider the effects on the accuracy of baseline determination of the geometric distribution of the satellites. Given the uncertainty in the measurement of interferometric phase delay, the determination of the corresponding uncertainty in the estimate of the baseline vector is non-trivial. The task is complicated largely because of the unknown, systematic, effects introduced by the neutral atmosphere. However, one pertinent question can be answered by an elementary analysis: What is the purely geometrical multiplying factor in the conversion of phase-delay uncertainty to baseline-component uncertainty? To obtain an answer, we used the following simplified expression for the interferometric phase delay obtained from observations of a satellite by two terminals (see, however, section III.A) : 


$$
\begin{aligned}
\tau_{12} \cong & \frac{1}{c}\left\{\left|\vec{s}-\vec{r}_{2}\right|-\left|\vec{s}-\vec{r}_{1}\right|\right\}+\tau^{c l} \\
\cong & \frac{1}{c}\left\{\left(\vec{r}_{2}-\vec{r}_{1}\right) \cdot \hat{s}+\frac{1}{4 s}\left[2\left(r_{2}^{2}-r_{1}^{2}\right)\right.\right. \\
& \left.-\left(\vec{r}_{2} \cdot \hat{s}^{2}+\left(\vec{r}_{1} \cdot \hat{s}\right)^{2}\right]\right\}+\tau^{c l} ; \\
& r \ll s,
\end{aligned}
$$

where $\vec{r}_{i}(i=1,2)$ and $\vec{s}$ are vectors, from the center of the Earth to the terminals and to the satellite, respectively. The analysis was based on the GPS configuration, with two satellites in each of the three orbital planes assumed to be crossing the equator at $t=0$. Baselines with $\left|\vec{r}_{2}-\vec{r}_{I}\right| \ll s$ were considered as an illustration; under these conditions, the term in brackets in Equation (2) can be ignored. However, the effects of parallax, due to the finite altitude of the satellites, must be considered and so $\hat{s}$ was calculated for the vector from the baseline midpoint to the satellite. Observations were assumed to be made of all satellites whose elevation angles, as viewed from the terminals, exceeded 10 deg.

From such observations, at each instant, the three components of the baseline vector and the epoch offset of the clock at one terminal with respect to that of the other, could be estimated and the standard errors in these estimates determined. The results from the analysis and related information on the geometry are presented in Figures 3 and 4 . Figure 3 is based on the terminals being placed at a north latituae of $40 \mathrm{deg}$ and at a longitude coincident at $t=0$ with an ascending node of one of the orbital planes of the satellites. In Figure 3a, we show the elevation angles as functions of time for all satellites visible from the terminals. Figure $3 \mathrm{~b}$ shows the results of the error analysis: the standard deviations in the estimates of the vertical and the two horizontal components of the baseline as functions of time. The standard deviations are given in units of the standard error in the determination of the interferometric phase delay. For either of the two horizontal components, the geometric multiplication factor is never more than 1.2 with its average value being about 0.8 . The multiplication factor is larger for the vertical component whose uncertainty depends more importantly on the total spread of the elevation angles. Thus, as can be seen in the $f$ igure, the multiplication factor is relatively large when the spread is small, and vice versa. For all three components, the standard errors as functions of time are discon-

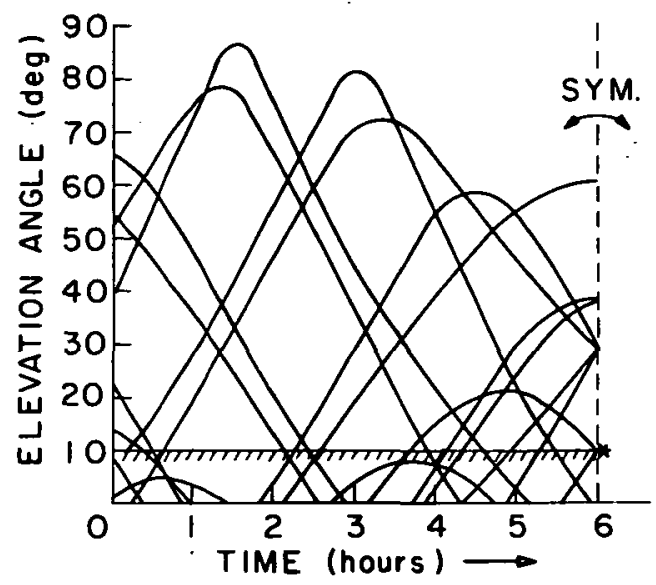

(a)

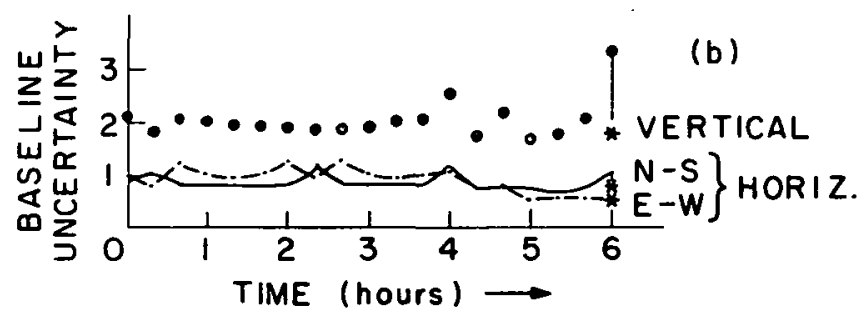

Fig. 3. Error analysis for Mighty MITES system.

(a) Elevation angles of satellites as seen from ground terminals at $40^{\circ} \mathrm{N}$. latitude. Observations are made of all satellites visible above $10^{\circ}$ elevation, except at $t=6$ hours, when the two satellites with elevations of $9.7^{\circ}$, indicated by the asterisk (*), are also observed.

(b) Standard errors of estimates of baseline vector components, computed at timeintervals of 20 minutes. Points plotted at these intervals have been connected by continuous lines for clarity, although actual "curves" have discontinuities when satellites cross $10^{\circ}$ elevation limit. Unit of baseline uncertainty is the standard error in measurement of interferometric phase delay, converted to equivalent path length. At time $=6$ hours, results are shown both for $10^{\circ}$ (regular symbols) and for $9.7^{\circ}$ (asterisks) elevation-angle cutoffs.

tinuous when a satellite passes either inside or outside the allowable elevation angle limit. We also note in the figure that if the elevation angle cutoff at $t=6 \mathrm{hr}$ were lowered by only $0.3 \mathrm{deg}$, the multiplication factor for the vertical component would drop nearly twofold, the total spread in the elevation angles then included being increased thereby from about 30 to $50 \mathrm{deg}$. Figure 4 contains the corresponding results for observations from terminals placed at the same longitude, but on the equator. 


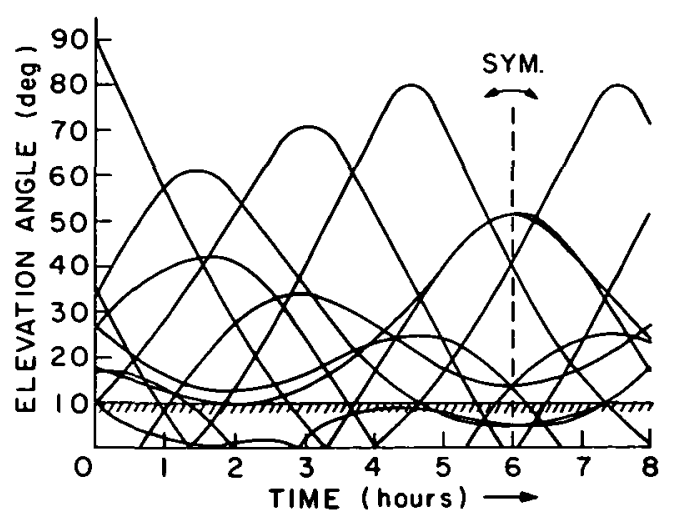

(a)

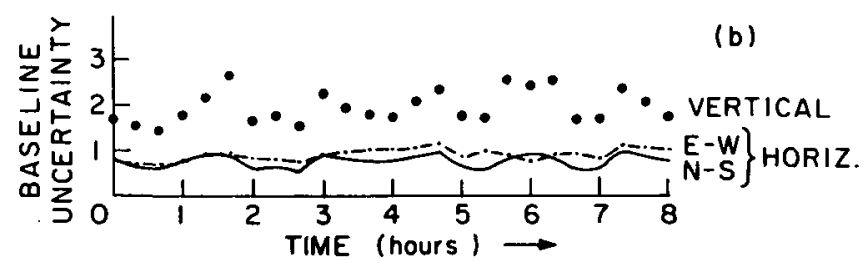

Fig. 4 (a) and (b). Same as Fig. 3, except that terminals are at the equator.

The above error analysis, as mentioned, ignores the effects of the troposphere and of the uncertainties in the orbits of the satellites. To obtain an indication of the magnitude of the effects of the troposphere, we repeated the analysis, but with the standard error for each observation equal to the cosecant of the elevation angle and with the elevation angle "cutoff" maintained at 10 deg. The results are summarized in Figure 5 . Here we see that the multiplication factors are larger, as expected, reaching up to 2.0 and 2.5 , respectively, for the horizontal components for terminals at $40 \mathrm{deg}$ and 0 deg latitude. The corresponding factors for the vertical component are both about 5.0, which serves to emphasize the importance of the observations being widely distributed in elevation angle, and, especially, being extended to high elevation angles.

In a further refinement, we could solve in addition for the common mode epoch error (see section III.A).

\section{Instrument}

For very closely spaced terminals the accuracy attainable in baseline determination could be limited by instrumental effects. For example, with a 100meter spacing of terminals, of some interest for surveying and for monitoring the effects of earthquakes (see section VI), the contribution to the error in baseline determination from the troposphere in good weather should be under $1 \mathrm{millimeter}$ and from the satelliteposition uncertainties under 0.05 milli- meters. Any instrumental effects of order 1 millimeter could, under these circumstances, degrade the system performance. Thus, it would be especially important for such applications to minimize the effects of ground reflections, as discussed in section II.C. With proper design and calibration, other sources of systematic errors in the instrument could probably be kept to a level of zbout $0.3 \mathrm{psec}$, equivalent to $0.1 \mathrm{~mm}$ in distance. The random errors, due to signal-to-noise limitations (Section II.C), could be reduced to this negligible level aiter less than ten minutes of integration. Should the ground reflections be the dominant contribution to the error, this fact would be disclosed in the slow variations with time of the estimate of the baseline, caused by the changing directions of the signals. These variations would also tend to repeat with a 12-hour period,' as indicated earlier.

A potential problem with current geodetic applications of VLBI concerns the determination of any changes with time of the length of the signal path through the antenna system. With the large structures now in use, one must account for deformations associated with changing winds, temperature, and gravity loads (see, for discussion, Rogers et al., 1978). However, for a miniature terminal with a dipole-type antenna such changes are negligible at the millimeter level due to the small size and rigidity of the antennas.

\section{Demonstration Experiment}

Could the feasibility of this system be demonstrated without the use of four or more satellites? The answer is yes, and the means are quite economical, too. A transmitter could, for example, be flown on each of four, small, private planes. More planes could be used for redundancy and, in addition or in replacement, one or more transmitters could be placed at suitable ground locations. For purposes of discussion, we assume that only four planes would be used. The planes could fly at altitudes of about 2 to $3 \mathrm{kilometers}$ and each could hold a tight, nearly circular, pattern of radius about 0.5 kilometers. The horizontal separation between planes could be about 5 to 6 kilometers to ensure good geometry without the use of excessively low elevation angles. The four planes could each be tracked in three dimensions by a simple set of four or more ground terminals, separated by, say, 50 to 100 meters and arranged suitably to ensure good geometrical relations. These ground terminals would be connected with phase- 
stable links, as in conventional radio interferometry, to eliminate clock epoch offsets as unknowns. Thus, the three, simultaneous, independent, measurements of interferometric phase for the signals from each plane would suffice to determine the three coordinates of the plane with respect to the reference frame defined by the four ground terminals. The relative surface locations of these ground terminals could easily be determined with the use of classical techniques to within a centimeter. Two, additional, "test" terminals could be placed near the center of the array of four terminals, about 10 or more meters apart from each other to reduce interference to a negligible level, and their vector separation determined from the signals received from the transmitters on the four planes. The epoch of the clock at one terminal would have to be known quite accurately a priori, or, alternatively, a fifth plane could be employed. The accuracy of the determination of the separation of the test terminals could be checked easily to the millimeter level with the use of simple, classical, geodetic techniques. The accuracy of the VLBI determination should be at the centimeter level, or below, depending on the accuracy of the location of the planes and on the relative separation of the various terminals. The direct tropospheric effects on this accuracy will be negligible, because of the small size of the array.

As an additional test, very low elevation angles could be used and the results compared as a function of terminal separation. It might also prove useful, with perhaps higher flying craft, to test extensively the effects of the troposphere on baseline determination in various locations and weather conditions.

Either of these simulations can be used to test just about all aspects of the proposed system, save for the technique to eliminate the effects of the ionosphere on the phase-delay measurements. Although only few may see the need for an actual test of this technique, because of our firmly based knowledge of the dispersive nature of a plasma, such a test is possible to perform. For example, one could use conventional VIBI observations of extragalactic radio sources; the center frequencies of the spectral regions sampled would be matched to the frequencies of the tones proposed for the satellite transmitters. The sampled regions of the spectrum would not be so narrow as for the tones because of the necessity to attain adequate signal-tonoise ratios. However this difference is inessential. The main difficulty with this test involves the need to develop suitable feed systems for the large antennas to cover the $\sim 1$ to $2 \mathrm{GHz}$ range effectively. Although observations of more than one source simultaneously will probably not be feasible in this test, the results from interleaved observations of a pair of sources can be used in much the same way as proposed for the Mighty MITES System.

\section{Applications}

There are a large number of potential applications of this interferometric system. We discuss some of these briefly.

\section{A. Earthquake Monitoring}

A primary use of the Mighty MITES would be to monitor the regional accumulation and release of strain. Their use would be most effective in regions around a fault such as the San Andreas where the terminals could be distributed widely, and yet densely, in the immediate vicinity of the fault and the data collected routinely. In effect, a seismic array could be set up, capable of measuring displacements with millimeter to centimeter precision in the 0 to $1 \mathrm{~Hz}$ part of the spectrum. The extraordinary time resolution of these measurements of baseline vectors and the high accuracy, especially for short baselines, should allow a very complete geometric characterization to be made of the crustal movements during actual earthquakes. Moreover, during earthquakes, any errors in our knowledge of satellite positions, which change slowly with time, would not significantly degrade the accuracy with which the changes in baseline vectors could be determined with the terminals.* These Mighty MITES should be sufficiently inexpensive to allow a dense net to be set up, and their ability to operate unattended should also cut down dramatically on the cost of the overall monitoring system, as indicated in section III. B.

Compact terminals could probably be designed to operate under rather extreme temperature and wind conditions; thus, such terminals could be placed at otherwise inaccessible locations, for example at the many Asian sites of geophysical interest where plates seem to be colliding and fracturing. Due to the use of low radio frequencies in our system, the signals could even penetrate through substantial snow and ice cover. However,

* Note, however, that during and immediately preceding and following earthquakes insufficient time would be available for averaging out short-term meteorological effects. 
signal reflections at the various interfaces might be devastating. In addition, under heavy snow or ice conditions, it might be necessary to use, say, radioisotope power to melt the snow and ice, and to operate the receiver and the transmitter, the latter to convey the data via a suitable satellite link to a data col-lection and processing center. Perhaps an acceptable global environmental impact statement could be adopted that would allow use of radioisotopes for such purposes.

In any event, the terminals could be built to survive winters and operate during the remainder of the year in normal fashion with, say, solar cells providing a source of power.

\section{B. Land Surveying}

These terminals could also be used for more conventional surveying. For example, for local surveying, over distances of a few kilometers or less, two or more terminals could be employed. The surveyor would merely have to set the terminals down at the positions whose vector separations are desired and, after the minute or so required for signal acquisition, baseline results could be obtained once per second. The surveyor would also require satellite-position information, a small microprocessor, and either a radio or a line link to each terminal to be able to determine these baselines. The needed computations could easily be done in real time and would thus allow the surveyor to complete his tasks virtually as fast as he could place the terminals at the desired positions. of course, observations could be continued by the surveyor as long as desired and the changes, or fluctuations, in the baseline vectors, as well as their running averages, could be monitored. Averaging largely removes the effects of short-term atmospheric fluctuations, caused, for example, by passing clouds.

Semi-permanent, self-contained, local arrays of terminals could also be used for a variety of purposes. As examples, we mention the monitoring of local crustal movements in the vicinity of nuclear plants, wells, pipelines, dams, mines, and rocket launching sites, and even the monitoring of oil rigs in the sea. In some cases, combination with gravity monitoring will yield a more powerful set of data for the determination of both crustal motions and changes in the mass underlying the area.

For purposes of: both two-way communication and supply of power, a direct electrical link to each terminal might be more suitable for fixed installations designed for long-term monitoring. If direct links are not convenient, small cassette recoraers could be used to store the data which could be collected intermittently.

\section{Navigation}

A number of navigational uses can be envisioned for the Mighty MITES. For example, they could be used aboard airplanes, boats, or land rovers. There should be no difficulty in the acquisition of satellite signals even aboard moving vehicles, provided the acceleration is not too high. Thus, a "one tenth g" acceleration could easily be tracked by the receiver. For significantly higher accelerations, a change in the design of the signal lock-on system might be required. The information on changes in vehicle position would also have to be transmitted to a central processor. Alternatively, the matching interferometric information on the signals received at some known, fixed site or sites could be transmitted to all vehicles from a central location and the determination of position carried out aboard each vehicle.

\section{Spacecraft Tracking}

The concepts involved in the Mighty MITES System could also be used in space applications. For example, with some modifications of the system to account for orbital motion, the vector separation between any two satellites could be monitored continuously provided both view the same five GPS satellites simultaneously. In fact, the GPS satellites themselves, if so equipped, could monitor their own vector separations! (However, one would have to take care in the frequency allocations to insure that the transmitted signals did not interfere with those received.) The reference system for the direction determinations would be an ensemble average; this (changing) system could be related to the extragalactic radio source reference frame through the previously-described differential VLBI measurements.

Another possibility is to use a set of these compact terminals at fixed locations to track any satellite equipped with an appropriate transmitter. With each terminal augmented by a suitab].? frequency standard, the accuracy a able with this system (see, for exarifie, Preston et al., 1972) might vej.1 be cotpetitive with that now obiainabie from laser tracking. In addition, the VLBJ system would have the advantages $n$ being all weather and having far less axpensivt ground equipment. (Even the transmitter may be less expensive than the satelititeborne optical corner reflectors.) .Satellite programs such as SEASAT and LANDSAT, some of whose scientific results depend 
importantly on the continuous determination of orbits with high accuracy, might especially benefit from use of this VLBI tracking system. The GPS si:tellites could, of course, also be tracked in this mode.

\section{E. Gravity Anomalies}

One possibly useful application of these concepts may be in the measurement of gravity anomalies on the Earth. Consider a pair of "drag-free" satellites separated by, say, 200 to $300 \mathrm{~km}$, and traveling in an orbit of that altitude. If each satellite were equipped with an appropriately modified Mighty MITES terminal to observe the GPS signals from the GPS satellites, sensitivity would exist to anomalies of the strength of $1 \mathrm{mgal}$ or greater, averaged over areas of about $200 \mathrm{~km}$ in linear extent. The limit on accuracy of this novel tracking system would be set by the (high-frequency) uncertainties in the knowledge of the orbits of the GPS satellites. Signalto-noise ratios and instrumental calibrations should easily be obtained which do not limit the system sensitivity above the stated spatial and temporal resolutions. There would be no contribution to the uncertainty by the tropospiere, unless the GPS satelitites were replaced in whole or in part by ground terminals.

Another variant of this general scheme would be to place a tone-transmitter on each of the low-orbit satellites and to place receivers on the GPS satellites. Three-dimensional tracking of the loworbit satellites could then also be accomplished in the GPS-defined reference frame, given suitable frequency standards aboard the GPS satellites. Again only high-frequency "noise" in the orbits of the GPS satellites would limit the sensitivity of the system to gravity anomalies.

\section{F. Miscellaneous}

One can also envision the Mighty MITES system being used for virtually instantaneous time transfer over transcontinental and intercontinental distances at about the few nanosecond level of accuracy. Here the accuracy would be limited primarily by the uncertainty in the knowledge of the positions of the GPS satellites.

other possible applications include monitoring the motion of icebergs and, for example, ice topography in locations such as Greenland and Antarctica. of course, during severe weather conditions, the operation of the system would be subject to the same constraints as mentioned in section VI.A.

We should emphasize that all of these potential applications must be considered in far greater detail before their utility can be assessed properly.

viI. Comparison with other space systems

How does this VLBI system compare with other "space" systems proposed for geodetic applications? We examine briefly five such possible systems as examples: the GPS, with and without modification of the satellites; a conventional VLBI system; a satellite Doppler positioning system; a ground-based laser system; and a spaceborne laser system.

\section{A. Global Positioning System}

The various options originally considered for GPS ground terminals were not intended to yield accuracy in position determination better than about 1 meter. A crucial question is: can the already planned GPS signals be utilized in other ways to yield substantially higher accuracies in position determination? Various possibilities can be envisioned. One possibility (MacDoran, 1978) would be to receive the GPS signals interferometrically as if they were random noise, as from extragalactic radio sources. Because of the strength of the GPS signals, even when treated as random noise, a l-meter diameter, transportable, antenna can be used with conventional VLBI receiving and data processing techniques. The estimated precision in baseline determination (MacDoran, 1978) is $2 \mathrm{~cm}$ from 1.5 hours of data collection for baselines $\$ 300 \mathrm{~km}$ in length. This system promises comparable accuracy to that claimed for the Mighty MITES system, but at the sacrifice of cost, simplicity, and time resolution.

Another possibility is to utilize knowledge of the pseudo-random noise GPS codes so that the effective noise bandwidth at the receiver is of the order of, say, ten Hertz instead of the order of ten Megahertz. One may then consider rapid determination of the interferometric group delay, at both the $\sim 1.2$ and the $1.6 \mathrm{GHz}$ GPS bands, with accuracy sufficient to determine the ionospheric contribution and to eliminate the $2 \pi$ ambiguities in interferometric phase delays. The two phase delays, one at: each of the two frequency bands, could then be combined to refine further the knowledge of the ionospheric contribution and to yield an accurate vacuumequivalent phase delay. (The requirements on group-delay accuracy are somewhat ameliorated for sites closelyenough spaced for the ionospheric effects on the interferometric observable to be negligible,) If this technique were viable, then the same accuracy in base- 
line determination could be achieved as with the Mighty MITES system. However, one important caveat which bears on the cost must be considered: the achievement of sufficient group delay accuracy to eliminate the phase delay ambiguity may entail considerable complication with such a narrow $(\approx 1$ \&) fractional bandwidth available at each GPS band. Although signal-to-noise ratios appear sufficient, systematic effects on the frequency dependence of the phase delays could be serious. As an oversimplified illustration, consider ground reflections of the signal that introduce an (erroneous) contribution to the slope of the phase of the signal as a function of frequency across the band. A difference in phase of only $1^{\circ}$, in opposite directions, at both ends of a $10 \mathrm{MHz}$ band, would introduce an error in group delay equivalent to a aisplacement of about $15 \mathrm{~cm} \mathrm{--} \mathrm{a}$ large fraction of the $\sim 20 \mathrm{~cm}$ wavelength at $1.6 \mathrm{GHz}$. To be more quantitative about the possible sources of such reflected signals, we note that the rootmean-square(rms) phase error due to, say, isotropic scattering by objects near the antenna is given approximately by

$$
\left.<\phi^{2}\right\rangle^{1 / 2} \simeq 10 \frac{\sigma^{1 / 2}}{R} \text { deg },
$$

where $\sigma$ is the cross section of the object, $R$ is its distance from the antenna, and both are measured in compatible units. We assume here that the receiving antenna is isotropic. Thus, an object such as a tree with a $2 \mathrm{~m}^{2}$ effective cross section, situated $15 \mathrm{~m}$ from the receiving antenna, would lead to a 1 deg rms phase error. To cut this figure to 0.1 deg would require the gain of the antenna to be $20 \mathrm{db}$ less in the direction of the scattered radiation than in the direction of the directly received signals. A simple antenna with such high directivity could not receive signals from all satellites simultaneously; hence the effects of instabilities of the frequency standard could not be eliminated simply by subtraction, as in the Mighty MITES system. A more stable, and presumably more expensive, standard would be needed. Alternatively, one could employ a phased-array antenna in either of two modes. In the simpler, but still rather elaborate mode, the pointing of this antenna could be cycled from satellite to satellite with the phase-locked loops that track the signals from any given satellite being gated in synchronism with this cycle. (The perjod of the cycle should be short compared with the time constants of the phase-locked loops.) In the second mode, the array could observe all visible satellites si- multaneously at the cost of multiple amplifiers and phase shifters for combining appropriately the outputs from the different elements of the array: We conclude that this problem of elimination of the $2 \pi$ ambiguity in phase with the planned GPS signals may require a rather expansive system to insure sufficient suppression of the effects of ground reflections (see also Section II.C).

In regard to systems, like ours, that involve use of modified GPS signals, one might enquire about non-interferometric schemes as well. We considered several such possibilities, for example a system in which the terminals received and transponded a ranging code to each satellite. None of these schemes looked as attractive as our interferometric system. Each had a serious flaw that seemed very costly to overcome.

\section{B. Conventional VLBI System}

A relatively large, transportable, conventional VLBI system that is used to observe extragalactic radio sources has several advantages over our compact terminal. First, since the observed signals are from extragalactic sources whose positions are virtually static, no orbit determinations are required and loss of accuracy in baseline determination with increasing baseline length is much more modest; second, the addition of watervapor radiometers to allow posisible achievement of higher accuracies in the estimate of tropospheric effects, is a relatively smaller complication for the larger system. The disadvantages of the larger VLBI system are partly economic: compact units, operating unattended, should prove far more cost effective with virtually unlimited numbers of such terminals usable simultaneously; and part technical: the temporal resolution achievable with the compact units should be at least three orders of magnitude better than with the larger system. Furthermore, determinations of baselines between all sites of interest simultaneously, which would be feasible only with compact terminals, would obviate the need for knowledge of polar motion and earthrotation variations to "tie" all intersite vectors to a common reference system.

\section{Satellite Doppler System}

The satellite Doppler system, which is used to determine positions on Earth from the Doppler shift of signals transmitted by satellites, has one distinct advantage over any VLBI system: each ground unit can be used independently to determine the position of any point on the Earth's surface in a suitably defined 
Earth-fixed reference system: However, this system has the apparent disadvantage that much more time of the order of many hours and perhaps days, may be required to determine the position of the receiver with accuracy comparable to that achievable between sites with VIBI; in fact, the attainable accuracy in relative position determination may still be severalfold worse than that attainabie with a VLBI system.

\section{Ground-Based Laser System}

Another system that could be used for monitoring crustal motions entails the use of highly-transportable lasers to observe the LAGEOS satellite from various sites (Bender et al., 1978). It is estimated that each such laser system would require several days per site to obtain a vector position with uncertainty at the centimeter level. Although the accuracy achievable with this system may be comparable to that expected with the proposed Mighty MITES system, the cost of each laser system will probably be more than a hundredfold greater and will require, on average, about a thousandfold longer time per position determination. The laser system would therefore suffer, relative to the Mighty MITES system, from the same difficulties discussed in connection with the conventional VLBI system. The main advantage of this laser system, as with every optical system, is its relative immunity to the effects of the water vapor in the troposphere (see Subsection E). Of course, optical systems are also freed from the complications entailed in the removal of ionospheric effects.

\section{E. Spaceborne Laser System}

The spaceborne laser system, which would measure the echo delays of signals reflected from arrays of optical corner cubes on the ground (Smith, 1978), has one main advantage with respect to the VI.BI system: The laser signals are about 40 times less sensitive than radio signals to the effects of atmospheric water vapor on the propagation times of the signals. The Mighty MITES system, on the other hand, has several distinct advantages over the laser system. First, with the Mighty MITES, baselines could be monitored continuously whereas, with lasers, the monitoring would be only intermittent, due both to weather interruption and to lack of continuous satellite coverage; measurements coincident with earthquakes, for example, might therefore be missed with the laser system. Second, the Mighty MITES system could be used effectively for long as well as for short baselines (although at
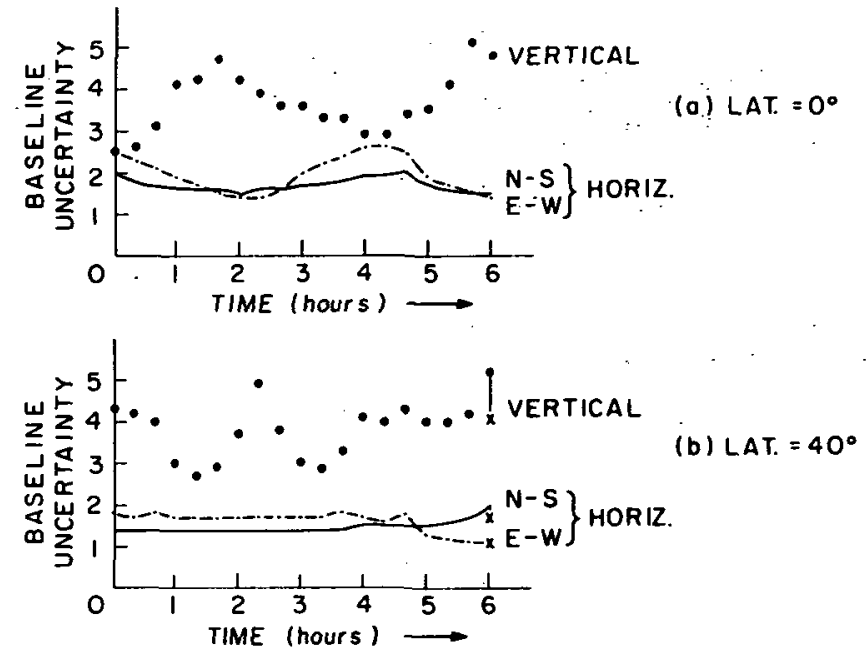

Fig. 5 (a) and (b). Same as Figs. 4 (b) and 3 (b), respectively, except that here the standard error in the measurement of the interferometric phase delay, instead of being unity, is equal to the cosecant of the elevation angle of the satellite.

greater expense because of the expected need to monitor the satellite orbits with larger VIBI systems), whereas the laser system, which may require a low orbit for the laser, would probably be considerably less effective in the determination of transcontinental baselines. Third, the spaceborne part of the Mighty MITES system is well within the state of the art and should have a lifetime of at least several years. By contrast, a laser system which can transmit pulses unattended and unmaintained at the required rates ior such a period has yet to be demonstrated. However, only one satellite at a time is required for the spaceborne laser system whereas many are required for the Mighty MITES system. (On the other nand, the requirements for pointing are far more severe for the laser than for the radio system.) With regard to the lifetime of the groundbased parts of the laser and radio systems, it is not clear which has the advantage: one must balance the maintenance of the electronic equipment, which may be capable of unattended performance for several years, against the erosive and obscuring effects on the corner cubes of wind-blow: dirt and rainborne dust.

The Mighty MITES receiver and electronics package could be placed underground, with only the antenna exposed to view. It is thus unclear whether corner cubes, which would be totally exposed above ground, or the radio terminals would be a greater attraction for souvenir hunters. 
Bender, P. L., et al., Tectonophysics (in press), 1978 .

Counselman, C. C., et al., Science, 178, 607,1972 .

Counselman, C. C., and H. Hinteregger, PrOC. I.E.E.E., 61, 478, 1973.

Counselman, C. C. , Annual Review of Astronomy and Astrophysics, 14, 197, 1976.

Elsmore, B., and M. Ryle, Mon. Not. Roy. Astron. Soc., 174, 411, 1976 .

International Telecommunications Union, "Radio Regulations" (International. Telecommunications Union, Geneva, 1968).

King, R. W. , et al., J. Geophys. Res., $81,6251,1976$.

MacDoran, P. F., presented at the International Symposium on the Use of Artificial Satellites for Geodesy and Geodynamics, Lagonissi, Greece, 29 May - 6 June, 1978.

Moran, J. M. ; and H. Penfield, Final Report, Contract NAS5-20975, 1976.

Murray, C. W., and J. W. Marini, GSFC Technical Memorandum, 1976.

Parkinson, B. W., "NAVSTAR Global Posttioning systems (GPS)", presented at the 1976 National Telecommunications Conference in Dallas, Texas (I.E.E.E. Catalog Number $76 \mathrm{CH} 1149-4 \mathrm{CSCB}$ ).

Preston, R., et.al., Science, 178, 407, 1972.

Robertson, D. S., et al., Recent Results of Radio Interferometric Determinations of a Transcontinental Baseline, Polar Motion, and Earth Rotation, in Proc. of IAU Symposium No. 82 (in press), 1979.

Rogers, A. E. E., Proc. I.E.E.E., 59, $1617,1971$.

Rogers, A. E. E., et al., J. Geophys. Res. , 83, 325, 1978.

Schaper, I. W. , et al., Proc. I.E.E.E., $58,272,1970$.

Smith; D. E., these Proceedings, 1978 . Whitney, A. R., et al., Radio Science, $11,421,1976$. 